\section{A implantação da rede de atenção psicossocial na Região Ampliada de Saúde Oeste de Minas Gerais-BR}

\section{The implementation of the psychosocial attention network in the extended Region of Health West of Minas Gerais-BR}

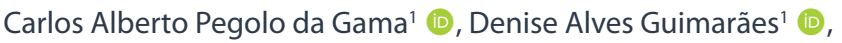 \\ Vívian Andrade Araújo Coelho' (D), Rafael Cotta Carvalho' (D), Cecília Godoi Campos² (D), \\ Agripina Maria de Souza Fraga² (D) \\ 'Universidade Federal de São João Del-Rei (UFSJ) - Divinópolis (MG), Brasil. \\ ${ }^{2}$ Núcleo de Atenção Primária à Saúde, Secretaria de Estado de Saúde (SRS), Regional Oeste Divinópolis, Divinópolis (MG), Brasil.
}

Como citar: Gama CAP, Guimarães DA, Coelho VAA, Carvalho RC, Campos CG, Fraga AMS. A implantação da rede de atenção psicossocial na Região Ampliada de Saúde Oeste de Minas Gerais-BR. Cad Saúde Colet, 2020;28(2):278-287. https://doi.org/10.1590/1414-462X202028020301

\section{Resumo}

Introdução: A criação da Rede de Atenção Psicossocial permitiu ampliar e organizar a oferta da atenção à saúde mental à população brasileira. Objetivo: Analisar a implantação da Rede de Atenção Psicossocial na Região Ampliada de Saúde Oeste de Minas Gerais. Método: Foi realizado estudo exploratório a partir de revisão de literatura, pesquisa documental, questionário aplicado a gestores e entrevistas com informantes-chave. Resultados: A Rede de Atenção Psicossocial possui 27 Centros de Atenção Psicossocial (CAPS) (13 CAPS I, 6 CAPS II, 1 CAPS III, 2 CAPS i, 3 CAPS ad II, 2 CAPS ad III), 12 Ambulatórios de Saúde Mental e 2 Residências Terapêuticas. A Estratégia de Saúde da Família está implantada em 86,62\% dos municípios e o Núcleo de Apoio à Saúde da Família em 62,96\%. Apresenta índice de 1,71 CAPS/100.000 habitantes, bem acima da média nacional. No entanto, $14,49 \%$ da população não possui acesso a este serviço. Conclusão: Constatou-se dificuldades na pactuação da Rede de Atenção Psicossocial nos municípios com menos de 15.000 habitantes, baixo índice de CAPS i e CAPS ad, insuficiência de CAPS III e escassa implementação de leitos em hospitais gerais.

Palavras-chave: saúde mental; serviços de saúde mental; serviços comunitários de saúde mental; pesquisa sobre serviços de saúde; reforma dos serviços de saúde.

\begin{abstract}
Background: The creation of the Psychosocial Care Network allowed the increase and organization of the provision of mental health care to the Brazilian population. Objective: This study aims to analyze the Psychosocial Care Network implantation process in the Western Health Extended Region of the State of Minas Gerais. Method: An exploratory study was carried out from literature review, documentary research, interviews with key informants and questionnaires that were applied to managers. Results: The Psychosocial Care Network has 27 Centers for Psychosocial Care (CAPS) (CAPS) (13 CAPS I, 6 CAPS II, 1 CAPS III, 2 CAPS i, 3 CAPS ad II, 2 CAPS ad III), 12 Mental Health Clinics and 2 therapeutic residences. The Family Health Strategy is implemented in $86.62 \%$ of the municipalities and the Family Health Support Unit in $62.96 \%$. It has an index of 1.71 CAPS / 100.000 inhabitants, above the national average; on the other hand $14.49 \%$ of the population does not have access to this service. Conclusion: It was observed difficulties in the agreement of Psychosocial Care Network in municipalities with less than 15.000 inhabitants, low CAPS i and CAPS ad index, CAPS III insufficiency and scarce beds implementation in General Hospitals have been found.
\end{abstract}

Keywords: mental health; mental health services; community mental health services; health services research; health care reform.

Trabalho realizado nos 54 municípios pertencentes a Região Ampliada Oeste de Saúde de (MG), Brasil.

Correspondência: Carlos Alberto Pegolo da Gama. E-mail: carlosgama@terra.com.br

Fontes de Financiamento: FAPEMIG - CHE - APQ-02530-16; Edital 01/2016 - Demanda Universal - O processo de implantação e a atuação dos profissionais do núcleo de apoio à saúde da família na atenção à saúde mental na região ampliada de saúde no oeste do estado de minas gerais. CNPq - Edital 002/2016/PROPE UFSJ (iniciação científica). Conflito de interesses: nada a declarar.

Recebido em: Out. 29, 2017. Aprovado em: Jul. 16, 2019

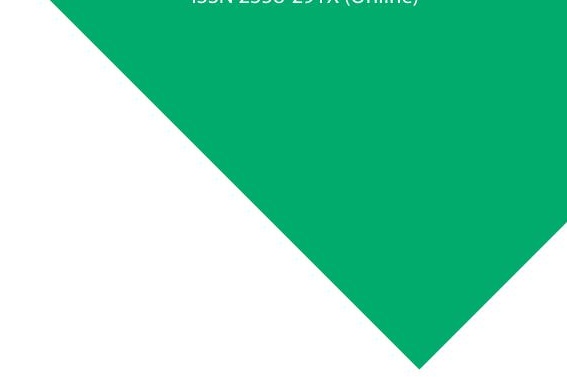

$-$ 


\section{INTRODUÇÃO}

A oferta de uma política de Saúde Mental para a população brasileira é uma tarefa complexa, tendo em vista o tamanho do país, as diversidades regionais e a complexidade inerente à área ${ }^{1,2}$. A Reforma Sanitária ${ }^{3}$ e a Reforma Psiquiátrica ${ }^{4}$ contribuíram nas últimas décadas para que esta política fosse aos poucos tornando-se realidade, sendo considerada, inclusive, modelo a ser seguido pelos países em desenvolvimento ${ }^{5}$. Esse processo caracterizou-se pela substituição de uma assistência hospitalocêntrica por um modelo territorializado, baseado em equipamentos comunitários $^{6}$. Constatam-se avanços ligados à legislação, diminuição das internações e construção de práticas clínicas ampliadas que introduzem o paradigma da atenção psicossocial ${ }^{1,6,7}$.

Em paralelo à construção deste novo modelo, o SUS como um todo foi se estruturando e consolidando suas propostas de regionalização da assistência, descentralização da gestão e financiamento da saúde pelos três entes federados ${ }^{8}$. Fazem parte desta construção a Estratégia de Saúde da Família (ESF) ${ }^{9}$; a criação do Núcleo de Apoio à Saúde da Família (NASF) ${ }^{10}$ e as Redes de Atenção à Saúde (RAS) ${ }^{11}$; o Decreto no $^{\circ} .508 / 11^{12}$ sobre a organização do SUS e as Redes de Atenção Psicossocial (RAPS) ${ }^{13}$. Como parte destas propostas, visando a superação da fragmentação então existente na assistência à saúde, houve liberação de fontes de financiamento para implantação de serviços considerados estratégicos para contratação de equipes e para processos de capacitação ${ }^{14}$.

Este processo de integração dos serviços de saúde é complexo, pois depende de contextos de cooperação e de uma ampliação da visão dos gestores para a compreensão dos problemas regionais, possibilitando a interlocução entre os diferentes níveis de governo e induzindo a organização ou reorganização de sistemas locais e regionais. Necessita também de linhas de financiamento e capacidade técnica das equipes ${ }^{15,16}$.

Apesar das dificuldades, é possível identificar diversas experiências no processo de criação destas redes espalhadas pelo território nacional e uma série de publicações nos periódicos especializados na área de saúde coletiva envolvendo temas relacionados à regionalização ${ }^{17,18}$, redes ${ }^{14,19}$, desigualdades regionais ${ }^{20}$, governança ${ }^{15,16}$, entre outros. Na área da saúde mental, observa-se que a proposta da RAPS estimulou iniciativas de estruturação de redes em diversas regiões e incentivou um processo de reflexão a respeito dos avanços e das fragilidades da Política Nacional de Saúde Mental 14,21,22.

A partir do cenário traçado, o objetivo do presente estudo é analisar a implantação da RAPS na Região Ampliada de Saúde Oeste de Minas Gerais (MG), produzindo informações relevantes para nortear as políticas públicas. Compreende-se que este diagnóstico da rede é fundamental, já que as recentes transformações no âmbito da saúde mental no Brasil vêm ocorrendo com escasso acompanhamento epidemiológico ${ }^{23,24}$.

\section{MÉTODO}

O estado de MG está dividido em 13 Regiões Ampliadas de Saúde ${ }^{12,25,26}$. O campo do presente estudo foi a Região Ampliada de Saúde Oeste que possui 54 municípios, distribuídos em 6 regiões de saúde (I- Divinópolis/Santo Antônio do Monte; II- Pará de Minas; III- Santo Antônio do Amparo/Campo Belo; IV- Formiga; V- Itaúna; VI- Bom Despacho). A população estimada é de 1.276 .557 habitantes para uma extensão territorial de $28.551,6 \mathrm{~km}^{2,25,26}$. Nesta Região Ampliada, 70\% dos municípios têm menos de 20.000 hab (Tabela1).

Foi realizado estudo exploratório, em parceria com a Superintendência Regional de Saúde Oeste da Secretaria Estadual de Saúde de MG (SRS/SES/MG), considerando as últimas 3 décadas (1990 - 2017). A coleta dos dados foi realizada no período de julho de 2016 a julho de 2017 a partir de diferentes fontes: 1) revisão de literatura nas bases de dados SCIELO, MedLine, PubMed; 2) pesquisa documental; 3) aplicação de questionário on-line de autopreenchimento e 4) entrevistas com informantes-chave.

A pesquisa documental considerou publicações oficiais do Ministério da Saúde(MS) ${ }^{1,69-13,27-30}$ sobre a normatização das redes e documentos da SES/MG $25,26,31$, sobre o processo de pactuação da RAPS. Os documentos foram lidos e as informações colocadas numa tabela em ordem cronológica orientando o processo descritivo de construção da RAPS. 
Tabela 1. Composição das regiões de saúde por porte populacional

\begin{tabular}{lcccccc} 
Região & População & $\begin{array}{c}\text { No de } \\
\text { municípios }\end{array}$ & $\begin{array}{c}\text { Pequeno } \\
\text { Porte } \mathbf{1}(\mathbf{A t e ́} \\
\mathbf{2 0 . 0 0 0} \mathbf{h a b})\end{array}$ & $\begin{array}{c}\text { Pequeno } \\
\text { Porte } \mathbf{2} \\
\mathbf{2 0 . 0 0 0} \\
\mathbf{5 0 . 0 0 0} \mathbf{h a b})\end{array}$ & $\begin{array}{c}\text { Médio Porte } \\
\mathbf{( 5 0 . 0 0 0 -} \\
\mathbf{1 0 0 . 0 0 0} \text { hab) }\end{array}$ & $\begin{array}{c}\text { Grande } \\
\text { Porte } \\
\text { (Maior que } \\
\mathbf{1 0 0 . 0 0 0} \text { hab) }\end{array}$ \\
\hline Região I & 469.432 & 13 & $6(46,15 \%)$ & $6(46,15 \%)$ & 0 & $1(7,69 \%)$ \\
\hline Região II & 238.959 & 8 & $5(62,5 \%)$ & $1(12,5 \%)$ & $2(25 \%)$ & 0 \\
\hline Região III & 205.455 & 13 & $11(84,61 \%)$ & $1(7,69 \%)$ & $1(7,69 \%)$ & 0 \\
\hline Região IV & 132.697 & 9 & $7(77,77 \%)$ & $1(11,11 \%)$ & $1(11,11 \%)$ & 0 \\
\hline Região V & 122.794 & 4 & $3(75 \%)$ & 0 & $1(25 \%)$ & 0 \\
\hline Região VI & 107.220 & 7 & $\mathbf{6 ( 8 5 , 7 1 \% )}$ & $1(14,28 \%)$ & 0 & 0 \\
\hline Região & $\mathbf{1 . 2 7 6 . 5 5 7}$ & $\mathbf{5 4}$ & $\mathbf{3 8 ( 7 0 , 3 7 \% )}$ & $\mathbf{1 0 ( 1 8 , 5 1 \% )}$ & $\mathbf{5 ( 9 , 2 5 \% )}$ & $\mathbf{1 ( 1 , 8 5 \% )}$ \\
Ampliada & & & & & & \\
\hline
\end{tabular}

Fonte: PDR-SUS/MG - Pop. ESTIMATIVA/IBGE/TCU-2016

O questionário on-line', composto por 27 questões fechadas foi elaborado em parceria com os profissionais da SRS a partir dos resultados obtidos nas pesquisas bibliográfica e documental. Este instrumento visou a coleta de dados primários relativos à composição e funcionamento da RAPS no nível municipal e regional, a presença e estrutura do NASF e sobre a organização da gestão da saúde mental nos municípios.

Os questionários foram enviados, por meio do software Surveymonkey ${ }^{\circledR}$, aos gestores responsáveis pela área de saúde mental de cada um dos 54 municípios pertencentes à região. Os respondentes foram convidados por meio eletrônico a participar da pesquisa e o retorno foi de $100 \%$. Esta alta taxa de retorno está relacionada com o apoio dos profissionais da SRS que têm contatos frequentes com os gestores dos municípios e nos ajudaram a monitorar as respostas, entrando em contato e solicitando a participação na pesquisa. Os dados quantitativos coletados foram tabulados, organizados em banco de dados, tabelas e trabalhados com técnicas da estatística descritiva.

Para complementar as informações obtidas por meio dos questionários foram identificados, com auxílio da SRS/SES/MG, 3 informantes-chave que participaram do processo de construção da RAPS na região analisada. Foram realizadas entrevistas direcionadas para complementar as informações faltantes sobre a implantação da rede. As informações qualitativas obtidas foram organizadas em categorias a partir do referencial da Análise de Conteúdo ${ }^{32}$. As categorias encontradas foram: Histórico da Saúde Mental na Região e níveis de estruturação da RAPS (Atenção Básica, Atenção Especializada, Atenção Urgência e Emergência, Atenção Residencial de Caráter Transitório, Atenção Hospitalar e Estratégias de Desinstitucionalização).

A pesquisa foi aprovada pelo Comitê de Ética em Pesquisa Envolvendo Seres Humanos da Universidade Federal de São João Del Rei, Campus Centro Oeste (CAAE: 77798217.1.3001.5091). Todos os participantes assinaram o Termo de Consentimento Livre e Esclarecido.

\section{RESULTADOS}

Como mencionado anteriormente, os resultados apresentados foram produzidos a partir das diversas estratégias de coleta propostas pela pesquisa. Os dados quantitativos que compõe as Tabelas 2 e 3 tiveram como fonte principal o questionário eletrônico. No entanto, após esta coleta primária, eles foram confrontados com documentos produzidos pela gestão estadual e revisados pelos informantes-chave durante as entrevistas. Os dados qualitativos tiveram origem em artigos que descrevem a história da saúde mental na região e nas entrevistas com

O questionário aplicado encontra-se disponível aos interessados mediante contato com o autor correspondente do presente artigo. 
Tabela 2. Dispositivos de atenção psicossocial. Minas Gerais - MG, 2014.

\begin{tabular}{|c|c|c|c|c|c|c|c|c|c|c|c|c|c|c|}
\hline Região & 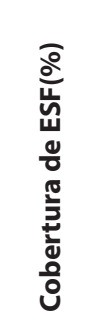 & 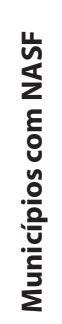 & U & 高 & $\frac{\overline{\bar{n}}}{\frac{\alpha}{\delta}}$ & $\begin{array}{l}\overline{\bar{n}} \\
\frac{a}{\mathbb{s}}\end{array}$ & $\frac{\bar{n}}{\frac{1}{\delta}}$ & 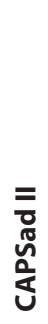 & $\begin{array}{l}\equiv \\
\bar{\delta} \\
\widetilde{N} \\
\frac{\alpha}{\delta}\end{array}$ & 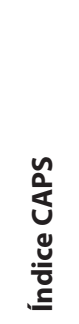 & 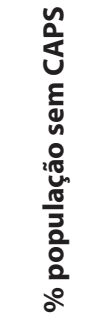 & 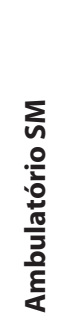 & $\stackrel{\frac{5}{n}}{\stackrel{n}{n}}$ & 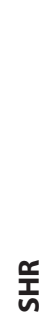 \\
\hline Região I & 67,41 & 6 & 0 & 5 & 0 & 1 & 0 & 0 & 1 & 1,17 & 14,54 & 5 & 1 & 1 \\
\hline Região II & 77,75 & 4 & 1 & 1 & 2 & 0 & 0 & 2 & 0 & 1,88 & 11,36 & 2 & 1 & 0 \\
\hline Região III & 99,07 & 11 & 0 & 3 & 1 & 0 & 2 & 0 & 1 & 2,67 & 26,36 & 2 & 0 & 0 \\
\hline Região IV & 92,78 & 8 & 0 & 2 & 1 & 0 & 0 & 0 & 0 & 1,51 & 12,97 & 1 & 0 & 0 \\
\hline Região Ampliada & 86,62 & 34 & 2 & 13 & 6 & 1 & 2 & 3 & 2 & 1,71 & 14,49 & 12 & 2 & 1 \\
\hline
\end{tabular}

Tabela 3. Porcentagem do total de internações psiquiátricas, no ano de 2015 , de pacientes provenientes da Região Ampliada Oeste de MG por tipo de hospital e município de sua localização. Minas Gerais - MG, 2015.

\begin{tabular}{|c|c|c|c|}
\hline \multicolumn{2}{|c|}{ Hospitais Psiquiátricos } & \multicolumn{2}{|c|}{ Hospitais Gerais } \\
\hline Hospital & $\begin{array}{c}\text { Total de } \\
\text { internações (\%) }\end{array}$ & Hospital & $\begin{array}{c}\text { Total de } \\
\text { internações (\%) }\end{array}$ \\
\hline $\begin{array}{l}\text { Clínica São Bento Menni } \\
\text { (Divinópolis) }\end{array}$ & 62,6 & Hospital São Luiz (Formiga) & 6,5 \\
\hline $\begin{array}{l}\text { Hospital Galba Veloso } \\
\text { (Belo Horizonte) }\end{array}$ & 7,1 & $\begin{array}{l}\text { Hospital São João de Deus } \\
\text { (Divinópolis) }\end{array}$ & 5,6 \\
\hline \multirow{3}{*}{$\begin{array}{l}\text { Instituto Raul Soares (Belo } \\
\text { Horizonte) }\end{array}$} & \multirow{3}{*}{$1,9 \%$} & $\begin{array}{c}\text { Hospital Nossa Senhora do } \\
\text { Brasil (Bambuí) }\end{array}$ & 5,3 \\
\hline & & Santa Casa (Arcos) & 2,3 \\
\hline & & Outros & 8,7 \\
\hline Total & 71,6 & Total & 28,4 \\
\hline
\end{tabular}

os informantes-chave que auxiliaram na compreensão do processo de implantação da RAPS em seus diversos níveis, na sua dinâmica e na identificação dos pontos mais vulneráveis das redes.

A Tabela 2 apresenta os principais componentes da RAPS na Região Ampliada:

No componente Atenção Básica, observa-se uma cobertura de ESF de $86,62 \%{ }^{28}$ e de NASF 62,9\%. Apenas 2 municípios possuem Centros de Convivência e a região não possui Equipe de Consultório de Rua implantada.

Com relação à Atenção Especializada, a região possui 1,71 CAPS/100.000 habitantes. A porcentagem da população que não tem referência de CAPS é de aproximadamente $15 \%$, distribuída em 24 municípios de pequeno porte. A região III, que tem a maior cobertura de ESF $(99,07 \%)$ e o maior índice de implantação de CAPS $(2,67)$, apresenta também o maior percentual 
de população sem retaguarda deste serviço (26,36\%). No tocante à assistência aos usuários de álcool e/ou outras drogas, foram implantados 3 CAPSad II e 2 CAPSad III. Na região III, o CAPSad III de Oliveira cobre 9 dos 13 municípios, o que representa aproximadamente $55 \%$ da população da região. Na região I, o CAPS ad III atende somente o município de Divinópolis e na região II, os CAPSad II de Pará de Minas e Nova Serrana atendem somente a população dos municípios. A respeito da assistência psicossocial infantojuvenil, a região III é a única que possui CAPSi, com 2 unidades que atendem a todos os municípios desta região de saúde.

No componente Atenção de Urgência e Emergência, a região possui 5 Unidades de Pronto Atendimento (UPA) implantadas e habilitadas pelo MS, uma UPA em funcionamento, mas não habilitada e uma UPA em construção. Em junho de 2017, o Serviço de Atendimento Móvel de Urgência (SAMU) iniciou-se na Região Ampliada. Este serviço conta com 31 veículos, sendo 07 Unidades de Suporte Avançado (USA) e 24 Unidades de Suporte Básico (USB).

Com relação à Atenção Hospitalar, foi encontrado somente um leito especializado em hospital geral, no município de Cláudio. Não foram encontradas enfermarias especializadas em hospital geral. A Clínica São Bento Menni (Divinópolis) era o local de referência para a internação psiquiátrica dos pacientes desta região. No ano de 2015, este hospital realizou 553 internações psiquiátricas, correspondendo a $62,6 \%$ de todas as internações psiquiátricas hospitalares dos pacientes da região. Em 2017, ele encerrou os atendimentos à clientela proveniente do SUS. Ainda no ano de 2015, outros hospitais foram responsáveis por um pequeno número de internações (Tabela 3). Nota-se que a maioria dos pacientes que chegaram a ser internados foram provenientes de regiões desprovidas de CAPS III.

Com relação aos Serviços de Atenção em Regime Residencial, a região conta com aproximadamente 60 Comunidades Terapêuticas. No componente Estratégias de Desinstitucionalização encontrou-se apenas 2 Serviços Residenciais Terapêuticos (SRT). A região conta ainda com 12 Ambulatórios de Saúde Mental (ASM) em funcionamento. Destes, 5 estão em municípios com menos de 20.000 habitantes e possuem além do ambulatório uma equipe de NASF. Os outros estão em municípios maiores que possuem CAPS.

\section{DISCUSSÃO}

Até a década de 1980, os serviços de saúde mental da região pesquisada estavam limitados aos ambulatórios e hospitais psiquiátricos. Os primeiros equipamentos substitutivos surgiram no início da década de 1990. No entanto, somente a partir da promulgação da Lei n 10.216 , em $2001^{6}$, é que esse processo começa a ganhar contornos mais definidos.

Em 2011 o MS institui a RAPS ${ }^{13}$. Já em 2012 a SES/MG deu início ao processo de pactuação das RAPS em cada uma das Regiões Ampliadas do estado ${ }^{25,26}$. Na Região Ampliada Oeste, a SRS/SES/MG de Divinópolis coordenou o processo que foi discutido na comissão SES/COSEMS (Conselho de Secretarias Municipais de Saúde de Minas Gerais) e, após análise de necessidades de cada município e cada região de saúde, definiu-se o desenho de rede e os serviços que deveriam ser implantados.

Conforme apontado, atualmente o índice de CAPS/100.000 habitantes $(1,71)$ na região é superior à média estadual $(0,95)$ e nacional $(0,76)$. Tal fato sinaliza avanço quanto à implantação da política de saúde mental' na região. Todas as regiões de saúde apresentam bons índices. No entanto, é possível perceber a persistência de municípios que não possuem CAPS implantado e que também não possuem uma referência para momentos de crise ${ }^{33}$. Percebem-se dificuldades na concretização de pactuações intrarregionais para compartilhamento de serviços relacionadas a uma intrincada configuração de atores, ações e interesses. Estudos demonstram que estas dificuldades estão presentes em todo o território nacional ligadas, muitas vezes, a uma lógica de disputas de recursos entre os entes governamentais e de subordinação a interesses privados ${ }^{16,17}$.

O CAPS III existente na região está localizado em Divinópolis, que é o único município de grande porte da Região Ampliada. A modalidade III indica funcionamento integral (24h) todos os dias da semana, incluindo finais de semana e feriados, e capacidade para acolhimento noturno quando necessário ${ }^{13,29}$. A ausência de CAPS III no restante da Região Ampliada pode 
ser consequência do custo maior deste equipamento e da falta de parcerias regionais mais estruturadas, impactando no manejo clínico do paciente em crise ${ }^{34}$.

Com relação à questão do álcool e outras drogas, o MS propõe, a partir de 2003, uma série de ações de prevenção e redução de danos, tendo como principal dispositivo de atenção os CAPSad. Diante disso, entende-se que a atenção regional ainda é frágil, com cobertura insuficiente, em consonância com a realidade nacional ${ }^{34}$. Apesar de contrastarem com as propostas do atual modelo assistencial, as comunidades terapêuticas são viabilizadas pelo governo de MG, desde 2013, por meio do programa Aliança pela Vida ${ }^{24}$. Como no restante do país, essas entidades são, em sua maioria, privadas, de caráter religioso e trabalham baseadas no princípio da abstinência. Diante desta opção, incluída na RAPS pelo governo federal desde 2011, muitos municípios da região optam por sua retaguarda ao invés de investir nos CAPS ad ${ }^{35,36}$.

A respeito da assistência psicossocial infantojuvenil, o baixo índice de CAPSi na Região Ampliada é reflexo da realidade brasileira que possui uma rede insuficiente e desigualmente distribuída majoritariamente em grandes centros. Estima-se que a prevalência brasileira de transtornos psiquiátricos em crianças e adolescentes está entre $7 \%$ e $24,6 \%$, sendo que entre $4 \%$ e 7,3\% demandam intervenção clínica ${ }^{37}$.

Os ASMs foram criados na região a partir da década de 1960 como porta de entrada dos hospitais psiquiátricos. Ao longo dos anos, com as mudanças propostas pela Reforma Psiquiátrica, vários municípios optaram por fechar os ambulatórios ${ }^{38}$. Entretanto, percebe-se que para os municípios menores, que não possuem CAPS, eles ainda desempenham um papel importante, pois frequentemente é onde está localizado o único psiquiatra do município. Neste ponto, diversos atores da rede temem um vazio assistencial caso o ASM seja fechado. Por outro lado, nas localidades onde essa instituição persiste, o acompanhamento dos transtornos menos severos também acaba sendo encaminhado para elas ao invés de serem manejados pela ESF conforme proposto pelas diretrizes nacionais ${ }^{39}$. Nos municípios maiores da região que possuem CAPS, os ASMs atendem sobretudo aos usuários com transtornos leves em consultas individuais de psiquiatria e psicologia apresentando imensas filas de espera e baixa resolutividade. Além disso, os ASMs mantém pouca ou nenhuma integração com os outros serviços da rede. Percebe-se a necessidade de aprofundamento desta discussão, repensando o papel dos ambulatórios na RAPS de forma a promover a integração dos ASMs aos demais serviços da RAPS e melhorar a retaguarda da ESF aos casos de saúde mental ${ }^{39}$.

Os SRTs são casas inseridas na comunidade para usuários que não possuem laços familiares ou suporte social e constituem a principal estratégia de desinstitucionalização para egressos de internações psiquiátricas de longa duração ${ }^{30}$. Na região estudada percebem-se poucas SRTs implantadas, sendo que em 2012, no plano de Ação de Saúde Mental do Estado foi pactuada a implantação de mais 2 serviços, mas estes não foram concretizados. Assim, a ausência de SRT nas regiões de saúde pode comprometer o eixo da desinstitucionalização proposto pela RAPS.

Com relação à atenção básica, a região possui cobertura média superior à nacional $(62,43 \%)^{28}$. Em 2003, o MS inicia o processo de inclusão das ações de saúde mental na atenção básica. O modelo propõe uma rede de cuidados articulada ao território com parcerias intersetoriais ${ }^{27}$. A proposta do NASF e das ações de matriciamento pelo MS em 2008 têm como objetivo ajudar neste processo. Entretanto, percebe-se que há dificuldade por parte dos gestores da região em operacionalizar este tipo de proposta, seja por deficiência no planejamento, por falta de recursos financeiros ou mesmo por falta de profissionais capacitados. Em muitos municípios, foi observada a utilização dos profissionais do NASF na assistência, demonstrando que a simples inclusão de profissionais especialistas em saúde mental nas ESF não produz as mudanças propostas ${ }^{40}$.

Com relação ao atendimento das urgências e emergências psiquiátricas e à atenção hospitalar, observou-se que, nos pequenos municípios que não contam com uma UPA instalada, as portas hospitalares de atenção à urgência e/ou a atenção básica responsabilizam-se pelos primeiros atendimentos de todos os tipos de urgência médica. Com relação aos casos de saúde mental, após os primeiros atendimentos nestes equipamentos, os pacientes, de acordo com as diretrizes da RAPS, deveriam ser encaminhados para o serviço de saúde mental mais próximo. Entretanto, observou-se que na região analisada, essas pactuações intermunicipais encontram-se 
incipientes, levando os municípios a manterem o paciente em atendimento ambulatorial de maneira precária no próprio território ou tentarem o encaminhamento dos casos graves para internação em hospital psiquiátrico através do sistema de regulação estadual (SUS fácil).

Segundo a portaria de regulamentação da RAPS, o hospital psiquiátrico pode ser acionado enquanto o processo de implantação e expansão da rede ainda não se apresenta suficiente. $\mathrm{Na}$ análise dos dados desta região observa-se, entretanto, que apesar do crescimento da oferta de serviços comunitários na região, a demanda para internações psiquiátricas hospitalares continua existindo. Por exemplo, em consulta realizada em 26 de abril de 2017 ao sistema de regulação do SUS fácil, existiam 75 pacientes da região aguardando vaga para internação na Clínica São Bento Menni.

De acordo com a proposta atual, a criação de leitos em hospitais gerais seria a estratégia adequada para lidar com essas situações mais complexas que ultrapassam a capacidade resolutiva dos CAPS. Entretanto, conforme demonstrado anteriormente, a região analisada conta com somente um leito implantado. Todavia, na análise dos dados encontrou-se um dado interessante: alguns hospitais gerais da região acabam internando estes usuários apesar de não terem os leitos específicos habilitados. Pode-se hipotetizar que este fato seja devido à busca de soluções locais para os momentos de crise, tendo em vista o tempo de espera para internação pelo SUS fácil e as escassas pactuações para compartilhamento de CAPS na região.

Para melhor esclarecimento deste ponto, fatores que não foram diretamente analisados neste trabalho merecem ser pesquisados, como, por exemplo, o motivo da não implantação dos leitos de saúde mental em hospitais gerais, a despeito do incentivo financeiro oferecido pelo governo federal, o modo de acolhimento destes usuários nas UPAs, o funcionamento dos CAPS e NASF em municípios de pequeno e médio porte, as particularidades de cada rede analisada, além das dificuldades na articulação dos dispositivos em rede. De toda forma, o pequeno número de CAPS III associado à quase inexistente implementação de leitos em hospitais gerais constitui uma realidade regional preocupante, uma vez que manejo da crise fora dos hospitais psiquiátricos garante ao indivíduo a permanência em seu meio sociocultural e familiar, rompendo com a lógica segregativa ${ }^{34}$.

O baixo nível de implantação dos centros de convivência e a inexistência de ações de reabilitação psicossocial, como oficinas de trabalho, empreendimentos solidários e cooperativas sociais indicam fragilidade regional nas propostas mais concretas de inserção dos portadores de sofrimento mental na comunidade.

O presente estudo mostra um panorama geral da implantação da RAPS na Região Ampliada Oeste de MG e foi centrado principalmente na dimensão estrutural da rede. Desta maneira, este estudo não se propôs a fazer uma avaliação dos serviços oferecidos e nem do funcionamento da RAPS. Entretanto, o diagnóstico realizado permite uma visualização das potencialidades e entraves presentes na atenção em saúde mental em uma ampla região do estado de MG municiando os gestores para melhorias do sistema e possibilitando que pesquisas futuras possam aprofundar as análises realizadas.

Os dados aqui analisados demonstram uma necessidade regional de maior aprofundamento nas negociações intermunicipais para a implantação de uma rede integrada. Neste sentido, deve-se considerar que as RAS possuem um modelo complexo de implantação e operacionalização e que necessitam, para sua efetivação, da substituição das atuais disputas por recursos entre os entes governamentais por um pensamento sistêmico que vise a resolução de problemas em conjunto em prol de uma melhor assistência.

\section{REFERÊNCIAS}

1. Brasil. Ministério da Saúde. Saúde Mental em Dados - 12 [Internet]. Inf Eletrônico. 2015;10(12):48. Available from: www.saude.gov.br/bvs/saudemental

2. Mendes Á, Funcia FRO. SUS e seu financiamento. In: Marques RM, Piola SF, Roa AC, editors. Sistema de Saúde no Brasil: organização e financiamento. Rio de Janeiro: Associação Brasileira de Economia da Saúde. Brasília: Ministério da Saúde; 2016. p. 139-168.

3. Paiva CHA, Teixeira LA. Reforma sanitária e a criação do Sistema Único de Saúde: notas sobre contextos e autores. História. Ciênc Saúde. 2014;21(1):15-35. 
4. Hirdes A. A reforma psiquiátrica no Brasil: uma (re) visão. Cien Saude Colet. 2009;14(1):297-305. http:// dx.doi.org/10.1590/S1413-81232009000100036. PMid:19142334.

5. Jacob K, Sharan P, Mirza I, Garrido-Cumbrera M, Seedat S, Mari J, et al. Mental health systems in countries: where are we now? Lancet. 2007;370(9592):1061-77. http://dx.doi.org/10.1016/S0140-6736(07)61241-0. PMid:17804052.

6. Brasil. Ministério da Saúde. Lei no 10.216, de 6 de abril de 2001. Dispõe sobre a proteção e os direitos das pessoas portadoras de transtornos mentais e redireciona o modelo assistencial em saúde mental. Diário Oficial da União [Internet], Brasília, 09 de abril de 2001. [citado em 2017 Feb 10]. Disponível em: http:// www.planalto.gov.br/ccivil_03/LEIS/LEIS_2001/L10216.htm

7. Pereira EC, Costa-Rosa A. Problematizando a Reforma Psiquiátrica na Atualidade: a saúde mental como campo da praxis. Saude Soc. 2012;21(4):1035-43. http://dx.doi.org/10.1590/S0104-12902012000400020.

8. Conill EM, Fausto MCR. Integração dos profissionais em redes regionalizadas de serviços: sobre conceitos, estratégias e alguns impasses. Rev Tempus Actas Saúde Col. 2012;6(2):179-94.

9. Brasil. Ministério da Saúde. Portaria n².488, de 21 de outubro de 2011. Aprova a Política Nacional de Atenção Básica, estabelecendo a revisão de diretrizes e normas para a organização da Atenção Básica, para a Estratégia Saúde da Família (ESF) e o Programa de Agentes Comunitários de Saúde (PACS). Diário Oficial da União [Internet], Brasília, 24 de outubro de 2011. [citado em 2017 Feb 10]. Disponível em: http:// bvsms.saude.gov.br/bvs/saudelegis/gm/2011/prt2488_21_10_2011.html

10. Brasil. Ministério da Saúde. Portaria GM n 154, de 24 de janeiro de 2008. Cria os Núcleos de Apoio à Saúde da Família - NASF. Diário Oficial da União [Internet], Brasília, 04 de march de 2008; Seção 1. [citado em 2017 Feb 10]. Disponível em: http://bvsms.saude.gov.br/bvs/saudelegis/gm/2008/prt0154_24_01_2008.html

11. Brasil. Ministério da Saúde. Portaria n 4.279, de 30 de dezembro de 2010. Estabelece diretrizes para a organização da Rede de Atenção à Saúde no âmbito do Sistema Único de Saúde (SUS). Diário Oficial da União [Internet], Brasília, 30 de dezembro de 2010. [citado em 2017 Feb 10]. Disponível em: http://bvsms. saude.gov.br/bvs/saudelegis/gm/2010/prt4279_30_12_2010.html

12. Brasil. Ministério da Saúde. Decreto n 7.508, de 28 de junho de 2011. Regulamenta a Lei n 8.080, de 19 de setembro de 1990, para dispor sobre a organização do Sistema Único de Saúde - SUS, o planejamento da saúde, a assistência à saúde e a articulação interfederativa, e dá outras providências. Diário Oficial da União [Internet], Brasília, 29 de junho de 2011. [citado em 2017 Feb 10]. Disponível em: http://www. planalto.gov.br/ccivil_03/_Ato2011-2014/2011/Decreto/D7508.htm

13. Brasil. Ministério da Saúde. Portaria GM n 3.088 de 23 de dezembro de 2011. Institui a Rede de Atenção Psicossocial para pessoas com sofrimento ou transtorno mental e com necessidades decorrentes do uso de crack, álcool e outras drogas, no âmbito do Sistema Único de Saúde (SUS). Diário Oficial da União [Internet], Brasília, 26 de dezembro de 2011. [citado em 2017 Feb 10]. Disponível em: http://bvsms.saude. gov.br/bvs/saudelegis/gm/2011/prt3088_23_12_2011_rep.html

14. Trapé TL, Campos RO. Modelo de atenção à saúde mental do Brasil: Análise do financiamento, governança e mecanismos de avaliação. Rev Saude Publica. 2017;51(19):1-13.

15. Ribeiro PT, Tanaka OY, Denis J-L. Governança regional no Sistema Único de Saúde: um ensaio conceitual. Cien Saude Colet. 2017;22(4):1075-84. http://dx.doi.org/10.1590/1413-81232017224.28102016. PMid:28444035.

16. Albuquerque MV, Lima LD, Oliveira RAD, Scatena JHG, Martinelli NL, Pereira AMM. Governança regional do sistema de saúde no Brasil: configurações de atores e papel das Comissões Intergovernamentais. Cien Saude Colet. 2018;23(10):3151-61. http://dx.doi.org/10.1590/1413-812320182310.13032018. PMid:30365836.

17. Moreira MR, Ribeiro JM, Ouverney AM. Obstáculos políticos à regionalização do SUS: percepções dos secretários municipais de Saúde com assento nas Comissões Intergestores Bipartites. Cien Saude Colet. 2017;22(4):1097-108. http://dx.doi.org/10.1590/1413-81232017224.03742017. PMid:28444037.

18. Fernandes FMB. Regionalização no SUS: uma revisão crítica. Cien Saude Colet. 2017;22(4):1311-20. http:// dx.doi.org/10.1590/1413-81232017224.26412016. PMid:28444054.

19. Trapé TL, Campos RO, Da Gama CAP. Mental Health Network: a narrative review study of the integration assistance mechanisms at the Brazilian National Health System. Int J Health Sci (Qassim). 2015;3(3):45-53. http://dx.doi.org/10.15640/ijhs.v3n3a5.

20. Albuquerque MV, Viana ALÁ, Lima LD, Ferreira MP, Fusaro ER, lozzi FL. Desigualdades regionais na saúde: mudanças observadas no Brasil de 2000 a 2016. Cien Saude Colet. 2017;22(4):1055-64. http://dx.doi. org/10.1590/1413-81232017224.26862016. PMid:28444033. 
21. Fagundes HM Jr, Desviat M, da Silva PRF. Reforma Psiquiátrica no Rio de Janeiro: situação atual e perspectivas futuras. Cien Saude Colet. 2016;21(5):1449-60. http://dx.doi.org/10.1590/1413-81232015215.00872016. PMid:27166894.

22. Costa PHA, Colugnati FAB, Ronzani TM. Avaliação de serviços em saúde mental no Brasil: revisão sistemática da literatura. Cien Saude Colet. 2015;20(10):3243-53. http://dx.doi.org/10.1590/1413812320152010.14612014. PMid:26465864.

23. Onocko-Campos RT, Furtado JP. Entre a saúde coletiva e a saúde mental: um instrumental metodológico para avaliação da rede de Centros de Atenção Psicossocial (CAPS) do Sistema Único de Saúde. Cad Saude Publica. 2006;22(5):1053-62. http://dx.doi.org/10.1590/S0102-311X2006000500018. PMid:16680358.

24. Coelho VAA, Volpe FM, Diniz SSL, Silva EM, Cunha CF. Alteração do perfil de atendimento dos hospitais psiquiátricos públicos de Belo Horizonte, Brasil, no contexto da reforma da assistência à saúde mental. Cien Saude Colet. 2014;19(8):3605-16. http://dx.doi.org/10.1590/1413-81232014198.11922013. PMid:25119099.

25. Minas Gerais. Secretaria de Estado da Saúde. Deliberação CIB-SUS/MG no 1.979, de 18 de novembro de 2014. Aprova o ajuste do Plano Diretor de Regionalização - PDR-SUS/MG 2014. Diário Oficial do Estado de Minas Gerais [Internet], Belo Horizonte, 18 de novembro de 2015. [citado em 2017 Fev 10]. Disponível em: http://saude.mg.gov.br/images/documentos/Del1979-PDR2014.pdf

26. Minas Gerais. Secretaria de Estado de Saúde. Deliberação CIB-SUS/MG n 2.400, de 19 de outubro de 2016. Aprova a Política Estadual de Saúde Mental, Álcool e Outras Drogas de Minas Gerais. Diário Oficial do Estado de Minas Gerais [Internet] 2016. [citado em 2017 Fev 10]. Disponível em: http://www.saude. mg.gov.br/images/documentos/Del2400SaúdeMental.pdf

27. Brasil. Ministério da Saúde. Secretaria de Atenção à Saúde. Saúde Mental e Atenção Básica: o vínculo e o diálogo necessários. Inclusão das ações de saúde mental na atenção básica. Brasília: Ministério da Saúde; 2003. p. 1-7.

28. Brasil. Ministério da Saúde. Secretaria de Atenção à Saúde. Departamento de Atenção Básica. Cobertura Saúde da Família. Brasília: Ministério da Saúde; 2017.

29. Brasil. Ministério da Saúde. Portaria GM/MS no 336 de 19 de fevereiro de 2002. Dispõe sobre os Centros de Atenção Psicossocial - CAPS, para atendimento público em saúde mental, isto é, pacientes com transtornos mentais severos e persistentes em sua área territorial, em regime de tratamento intensivo, semi-intensivo e não-intensivo. Diário Oficial da União [Internet], Brasília, 20 de fevereiro de 2002; Seção 1. p. 22 [citado em 2017 Fev 10]. Disponível em: http://bvsms.saude.gov.br/bvs/saudelegis/gm/2002/ prt0336_19_02_2002.html

30. Brasil. Ministério da Saúde. Portaria n 3.090, de 23 de Dezembro de 2011(*). Altera a Portaria n 106/GM/ MS, de 11 de fevereiro de 2000, e dispõe, no âmbito da Rede de Atenção Psicossocial, sobre o repasse de recursos de incentivo de custeio e custeio mensal para implantação e/ou implementação e funcionamento dos Serviços Residenciais Terapêuticos (SRT). Diário Oficial da União [Internet], Brasília, 26 de dezembro de 2011; Seção 1, p. 233-234 [citado em 2017 Fev 10] Available from: http://bvsms.saude.gov.br/bvs/ saudelegis/gm/2011/prt3090_23_12_2011_rep.html

31. Minas Gerais. Secretaria de Saúde. Deliberação CIB-SUS/MG no 1.219, de 21 de agosto de 2012. Institui as Regiões de Saúde no âmbito do Sistema Único de Saúde (SUS) para o Estado de Minas Gerais, e dá outras providências. Diário Oficial do Estado de Minas Gerais [Internet] 2012; [citado em 2017 Fev 10]. Available from: https://www.saude.mg.gov.br/images/documentos/Deliberacao 1.219 - Regioes de Saude- 21.8.12.pdf

32. Bardin, L. Análise de conteúdo. Lisboa: Edições 70. 2006. (Obra original publicada em 1977).

33. Neves LA, Ribeiro JM. Consórcios de saúde: estudo de caso exitoso. Cad Saude Publica. 2006;22(10):220717. http://dx.doi.org/10.1590/S0102-311X2006001000027. PMid:16951893.

34. Pitta AMF. An assessment of Brazilian Psychiatric Reform: Institutions, actors and policies. Cien Saude Colet. 2011;16(12):4579-89. http://dx.doi.org/10.1590/S1413-81232011001300002. PMid:22124894.

35. Varela DSS, Sales IMM, Silva FMD, Monteiro CFS. Rede de saúde no atendimento ao usuário de álcool, crack e outras drogas. Esc Anna Nery. 2016;20(2):296-302.

36. Coelho VAA, Costa-Val A, da Silva RA, Cunha CF. Navegar é preciso, viver é (im) preciso. Estud Contemp da Subjetividade ECOS. 2016;6(1):99-112.

37. Garcia GYC, Santos DN, Machado DB. Centros de atenção psicossocial infantojuvenil no Brasil: distribuição geográfica e perfil dos usuários. Cad Saude Publica. 2015;31(12):2649-54. http://dx.doi.org/10.1590/0102311X00053515. PMid:26872240.

38. Santos YF, Oliveira IMFF, Yamamoto OH. O ambulatório de saúde mental no contexto da reforma psiquiátrica em Natal, RN. Psicol Argum. 2009;27(59):313-22. 
39. Damous I, Erlich H. O ambulatório de saúde mental na rede de atenção psicossocial: reflexões sobre a clínica e a expansão das políticas de atenção primária. Rev Saúde Coletiva. 2017;27(4):911-32. http:// dx.doi.org/10.1590/s0103-73312017000400004.

40. Tófoli LF, Fortes $S$. Apoio matricial de saúde mental na atenção primária no município de Sobral, CE: o relato de uma experiência. Sonare, Sobral. 2007;6(2):34-42. 